\title{
Development of Urinary Bladder Pre-Neoplasia by Schistosoma haematobium Eggs and Chemical Carcinogen in Mice
}

\author{
Bayissa Chala', Min-Ho Choi', Kyung Chul Moon², Hyung Suk Kim³, , Cheol Kwak³, Sung-Tae Hong,"* \\ ${ }^{1}$ Department of Parasitology and Tropical Medicine, Institute of Endemic Diseases, Seoul National University College of Medicine, Seoul 03080, \\ Korea; ${ }^{2}$ Department of Pathology and ${ }^{3}$ Department of Urology, Seoul National University College of Medicine, Seoul 03080, Korea
}

\begin{abstract}
Schistosoma haematobium is a biocarcinogen of human urinary bladder (UB). The present study investigated developing UB cancer mouse model by injecting $S$. haematobium eggs into the bladder wall and introduction of chemical carcinogens. Histopathological findings showed mild hyperplasia to epithelial vacuolar change, and high grade dysplasia. Squamous metaplasia was observed in the $S$. haematobium eggs+NDMA group at week 12 but not in other groups. Immunohistochemistry revealed significantly high expression of Ki-67 in urothelial epithelial cells of the S. haematobium eggs+BBN group at week 20. The qRT-PCR showed high expression of $p 53$ gene in S. haematobium eggs group at week 4 and S. haematobium eggs+BBN group at week 20. E-cadherin and vimentin showed contrasting expression in S. haematobium eggs+BBN group. Such inverse expression of E-cadherin and vimentin may indicate epithelial mesenchymal transition in the UB tissue. In conclusion, S. haematobium eggs and nitrosamines may transform UB cells into squamous metaplasia and dysplasia in correlation with increased expression of Ki-67. Marked decrease in E-cadherin and increase in p53 and vimentin expressions may support the transformation. The present study introduces a promising modified animal model for UB cancer study using S. haematobium eggs.
\end{abstract}

Key words: Schistosoma haematobium, bladder cancer, mouse, egg, N-nitrosodimethylamine, N-butyl n-(4-hydroxybutyl) nitrosamine

\section{INTRODUCTION}

Schistosoma haematobium has been classified as a class 1 carcinogen by International Agency for Research on Cancer (IARC) of the World Health Organization [1]. Available data suggest that squamous cell carcinoma (SCC) of urinary bladder (UB) arises from transformation of squamous metaplastic lesions, which occurs secondary to inflammation initiated by urinary schistosomiasis [2]. Previous studies reported that SCC accounts for up to $75-83 \%$ of bladder cancers in certain schistosomiasis endemic regions of the world $[3,4]$. In schistosomiasis endemic areas of Africa, the prevalence of SCC has been reported to be higher compared to conventional transitional cell carcinoma (TCC), especially in rural areas [5]. Felix et al. [6] reported that the occurrence of TCC of the bladder has

\footnotetext{
- Received 12 October 2016, revised 19 January 2017, accepted 1 February 2017.

*Corresponding author (hst@snu.ac.kr)

† Present address: Department of Urology, Dongguk University Ilsan Medical Center, Goyang 100326, Korea

(C) 2017, Korean Society for Parasitology and Tropical Medicine

This is an Open Access article distributed under the terms of the Creative Commons Attribution Non-Commercial License (http://creativecommons.org/licenses/by-nc/4.0) which permits unrestricted non-commercial use, distribution, and reproduction in any medium, provided the original work is properly cited.
}

supplanted SCC in Egypt following a major decline in the prevalence of UB schistosomiasis, which suggests the importance of schistosome-associated bladder cancer [7]. Recently, a retrospective review of 185 patients in north-western Tanzania reported 55.1\% of SCC whereas $40.5 \%$ TCC and $44.9 \%$ of all cancer cases were found to have Schistosoma eggs [8]. Similarly, a study from Angola reported a prevalence of $71.7 \%$ S. haematobium infection with $3.4 \%$ vesical tumor, classified as SCC [9].

Nweke et al. [10] reviewed that several studies overwhelmingly documented the epidemiologic and experimental association of the parasite with SCC of the UB cancer. The studies showed that chronic inflammatory processes induced by the eggs on the bladder wall generated toxic free radicals and $\mathrm{N}$ nitrosamines. The N-nitrosamines, including N-nitrosodimethylamine (NDMA), were classified by IARC as Group 2A carcinogenic compounds that can also be formed endogenously from the interaction of ingested nitrate or nitrite with secondary amines. The $\mathrm{N}$-nitrosamines are eventually activated by the $\mathrm{p}-450$ of the liver to genotoxic $\mathrm{N}$-nitroso compounds.

According to previous reports from animal experiments, noninvasive papillary and nodular TCC of the urinary bladder 
were observed in gibbons (Hylobates lar) and non-human primates like baboon (Papio sp.) when infected with $S$. haematobium with or without $\mathrm{N}$-nitroso chemicals $[11,12]$. Several studies reported that current UB cancer models in rodents rely on the administration of nitrosamines mainly N-butyl n-(4hydroxybutyl) nitrosamine (BBN) in drinking water, leading to onset of invasive carcinoma after 3-4 months of exposure $[13,14]$.

Recently, a new mouse model was developed for immune response studies by artificial injection of $S$. haematobium eggs into the UB wall [15]. Accordingly, egg deposition alone is sufficient to reproduce important aspects of urogenital schistosomiasis like bladder fibrosis, upregulation of IL-4, and various urothelial changes which are morphologically reminiscent of human urogenital schistosomiasis, even without the other life stages of the parasite [16].

In this context, there seems sufficient evidence for the initiation of bladder tumors by administering chemical carcinogens like BBN alone or BBN followed by N-nitroso-N-methylurea (NMU) in primates and rodents under experiments. However, there were no recent studies reporting the scientific association of artificial injection of $S$. haematobium eggs into the mouse UB wall accompanied with exogenous administration of NDMA for possible urothelial neoplastic development. Therefore, the present study investigated the separate and synergistic effects of $S$. haematobium eggs injection into the UB wall of mice and concurrent exogenous administration of NDMA and BBN. The present study also analyzed expressions of tumor suppressor and epithelial mesenchymal transition (EMT) marker genes to strengthen the evidence for development of bladder preneoplasia.

\section{MATERIALS AND METHODS}

\section{Ethical statement}

The animal experiment protocol was reviewed and approved by the institutional animal care and use committee (IACUC) of Seoul National University (SNU), Seoul, Korea (no. SNU-141126-2-2).

\section{Animals, parasites, and reagents}

Female ICR mice of 5 weeks old (average weight, $30 \mathrm{~g}$ ) on arrival were purchased from Orient Bio Inc. (Seongnam, Korea) and were randomly assigned to 6 groups. The mice were housed in biosafety level 2 (BSL-2) animal facilities. Eggs of $S$.

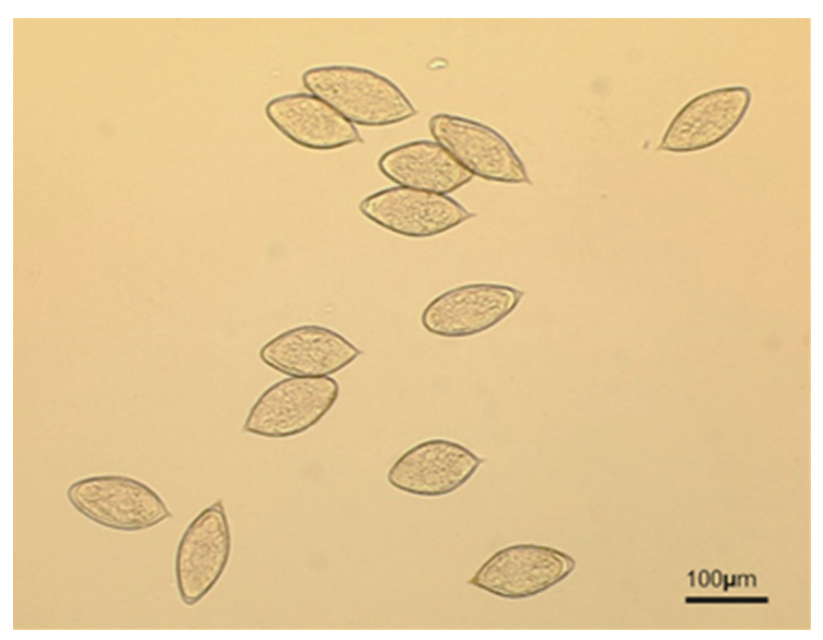

Fig. 1. S. haematobium eggs isolated from patients' urine samples.

haematobium were collected from patients in Sudan and isolated from urine samples in the laboratory of the Department of Parasitology and Tropical Medicine, SNU, Korea (Fig. 1). NDMA and BBN were purchased from Sigma-Aldrich (St. Louis, Missouri, USA) and stored undiluted in the dark at $-20^{\circ} \mathrm{C}$.

\section{Experimental design}

A total of 72 female ICR mice were randomly divided into 6 groups: Group 1 (control), Group 2 (S. haematobium eggs), Group 3 (NDMA), Group 4 (BBN), Group 5 (S. haematobium eggs+NDMA), and Group 6 (S. haematobium eggs+BBN). Group 1, 2, 5, and 6 mice underwent anesthesia by isoflurane and midline lower abdominal incision for the bladder exteriorization. About 1,000 S. haematobium eggs in $50 \mu \mathrm{l}$ saline was injected submucosally into the the anterior part of the bladder dome of mice of Group 2, 5, and 6, while Group 1 mice were injected with $50 \mu \mathrm{l}$ sterile saline. Both NDMA and BBN, once diluted in distilled water under chemical biohazard hood, were administered to the respective mice groups at $12.5 \mathrm{ppm}$ and $0.05 \%$ concentration, respectively, and provided to mice ad libitum throughout the experimental process. Two mice from each group were sacrificed after 4, 12, 20, 28, and 36 weeks post injection with $S$. haematobium eggs and NDMA or $\mathrm{BBN}$ treatment. The body weight of all mice was measured every week using an electronic balance.

\section{Histopathological and immunohistochemical analysis}

Following mouse sacrifice, the whole UB was harvested and divided into $2 / 3$ and $1 / 3$ sections for histopathology and mRNA expression, respectively. The 2/3 tissue was kept in neu- 
tral buffered formalin $10 \%(\mathrm{v} / \mathrm{v})$ for a few days until paraffin embedding and blocking for routine histology. The UB tissue was sectioned by $4-5 \mu \mathrm{m}$ in paraffin block and stained with routine hematoxylin and eosin (HE) solutions. Histological lesions and abnormalities of tissue sections were classified and staged according to standard histopathological examination [17]. Images were captured with a light microscope (Olympus CKX41 microscope, Tokyo, Japan) linked to a computer.

Immunohistochemical analysis of nuclear cell proliferation marker was performed using monoclonal rat anti-mouse Ki-67 antigen (Dako, Carpinteria, California, USA). Secondary biotinylated goat anti-mouse antibodies were used. Antibodies were diluted in the recommended antibody diluting buffer. The working dilutions and the final concentrations of the primary and secondary antibodies for anti-Ki-67 were 1:200 and $0.005 \mathrm{mg} / \mathrm{ml}$ and $1: 200$ and $0.0025 \mathrm{mg} / \mathrm{ml}$, respectively. The immunostained samples were compared among the groups and across the weeks of sacrifice.

\section{RNA extraction and cDNA synthesis}

The $1 / 3$ proportions of UB tissues of the respective sacrificed mice groups were preserved in liquid nitrogen for extraction of RNA. Total RNA was extracted using PureLink ${ }^{\mathrm{R}}$ RNA Mini kit (Invitrogen, Carlsbad, California, USA) according to the manufacturer's instructions. After quantitation by a NanoDrop spectrophotometer, up to $2 \mu \mathrm{g}$ RNA sample was reversely transcribed into CDNA according to the instructions provided by High-Capacity cDNA Reverse Transcription Kits (Applied Biosystems, Foster City, California, USA). The cDNA samples were kept at $-70^{\circ} \mathrm{C}$ until use.

\section{Analysis of gene expression by real-time PCR assay}

The resulting cDNA was amplified by TOPreal qPCR 2X PreMix (SYBR Green with high ROX) (Enzynomics, Daejeon, Korea) using a primer set specific for E-cadherin, $p 53$, vimentin, and GAPDH (reference gene) (Bionics, Seoul, Korea). The expression of the target genes was quantified by qRT-PCR. The primer design for the target genes was obtained from NCBI database as follows: (E-cadherin NM_009864.3): E-cadherin (forward, 5'-GATCCTGCTGCTCCTACTGT-3'), E-cadherin (reverse, 5'-GCTCAAATCAAAGTCCTGGTCT-3'), (p53 NM_001127233.1): p53 (forward, 5'-TGAAACGCCGACCTATCCTTA-3'), p53 (reverse, 5'-GGCACAAACACGAACCTCAAA-3'), (vimentin NM_011701.4): vimentin (forward, 5'-CCTTGACATTGAGATTGCCA-3'), vimentin (reverse, 5'-GTATCAACCAGAGGGAGTGA-3'), (GAPDH
NM_001289726.1): GAPDH (forward, 5'-GGTGAAGGTCGGTGTGAACG-3'), and GAPDH (reverse, 5'-GCGCTCCTGGAAGATGGTG-3') [18,19]. All comparative expression data were analyzed using Microsoft Excel 2010 version with a cycle threshold $(\mathrm{Ct})$ in the linear range of amplification adapted from Nature Protocols [20].

\section{Data analyses and statistics}

Data were analyzed using Microsoft Excel 2010 version and GraphPad Prism 5 (GraphPad Software, San Diego, California, USA). Comparisons of results were performed using a Student's $t$-test. $P$-value $<0.05$ was considered as significant.

\section{RESULTS}

\section{Body weight comparison}

From week 15 to 29 , a highly significant reduction (20.5\%) of body weight was observed in $S$. haematobium eggs $+\mathrm{BBN}$ (36.3 $\pm 7.2 \mathrm{~g} ; P<0.01)$ and $S$. haematobium eggs+NDMA (11.6\%) with weight loss $(40.3 \pm 8.5 \mathrm{~g} ; P<0.01)$ groups compared to the control group (45.6 $\pm 8.3 \mathrm{~g})$, also in NDMA group (42.7 \pm 9.1 ; $P<0.01)$, and BBN group $(42.2 \pm 7.3 ; P<0.01)$, but not in $S$. haematobium eggs group $(43.4 \pm 9.7 ; P=0.12)$.

\section{Histopathological findings}

HE staining of the present study revealed marked focal and dispersed tissue reactions and inflammatory aggregates mainly among BBN, S. haematobium eggs+NDMA and S. haematobium eggs+BBN groups starting from week 4 through week 20 (Fig. 2). However, tissues from the $S$. haematobium eggs or NDMA group showed no comparable histopathological changes. Similarly, ranges of histopathological anomalies like abnormal nuclear structure, including enlarged and pleomorphic cells and prominent nucleoli were observed among the same groups. Preneoplastic indicators, such as hyperplasia and dysplasia, were noticed in BBN, S. haematobium eggs+NDMA, and $S$. haematobium eggs+BBN groups, while squamous metaplasia and epithelial vacuolar change (black arrows) were exclusively observed in S. haematobium eggs+NDMA and S. haematobium eggs+BBN groups at week 12 and 20, respectively (Fig. 3). The urothelial epithelial cells which underwent hyperplasia and dysplasia presented more than 6 layers in comparison to the 2 to 3 layers (basal, intermediate and umbrella layers) seen in controls, S. haematobium eggs+NDMA groups. 

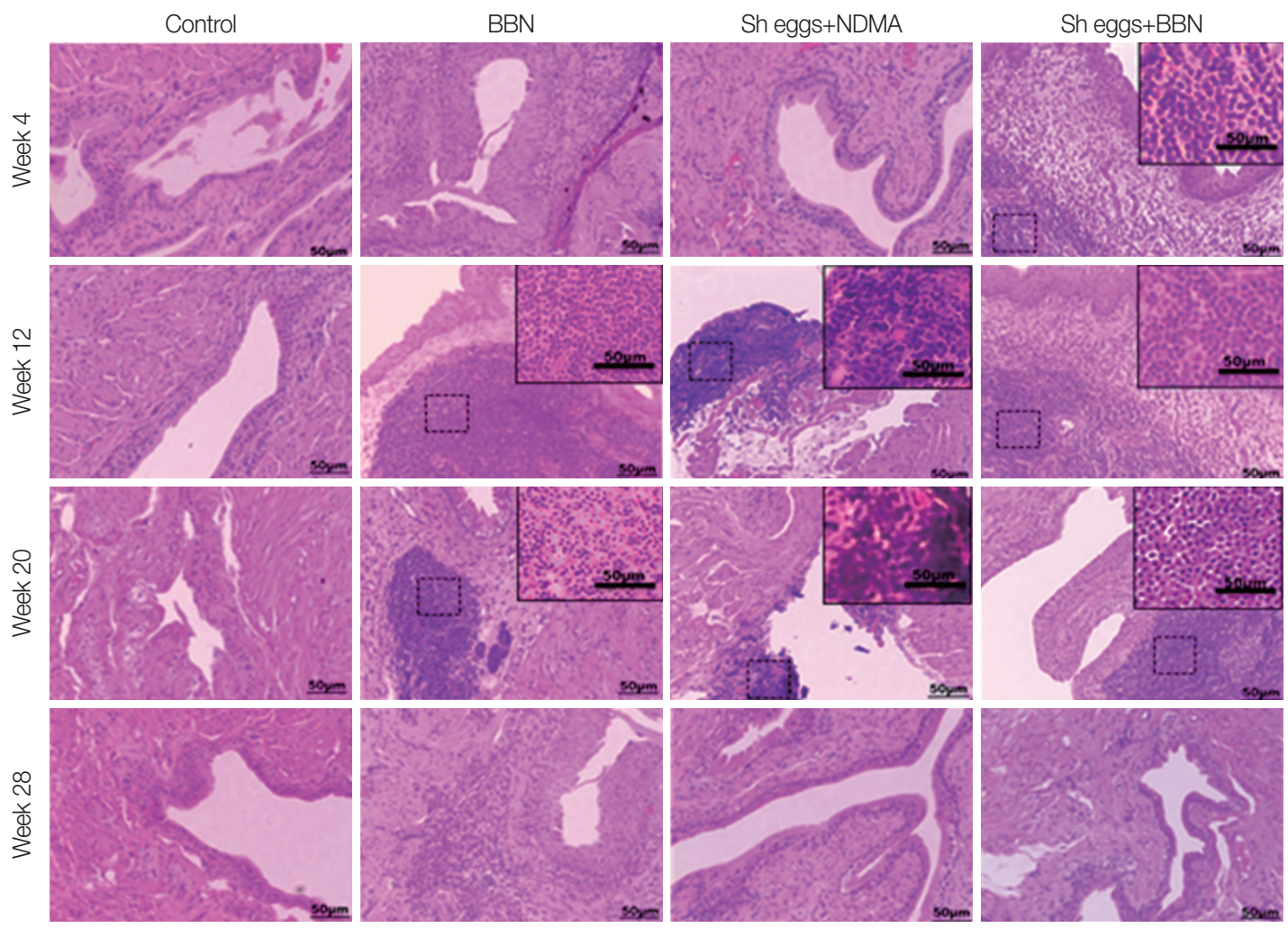

Fig. 2. Tissue sections of mouse urinary bladder showing various tissue reactions (darker inflammatory aggregates) (HE stain).
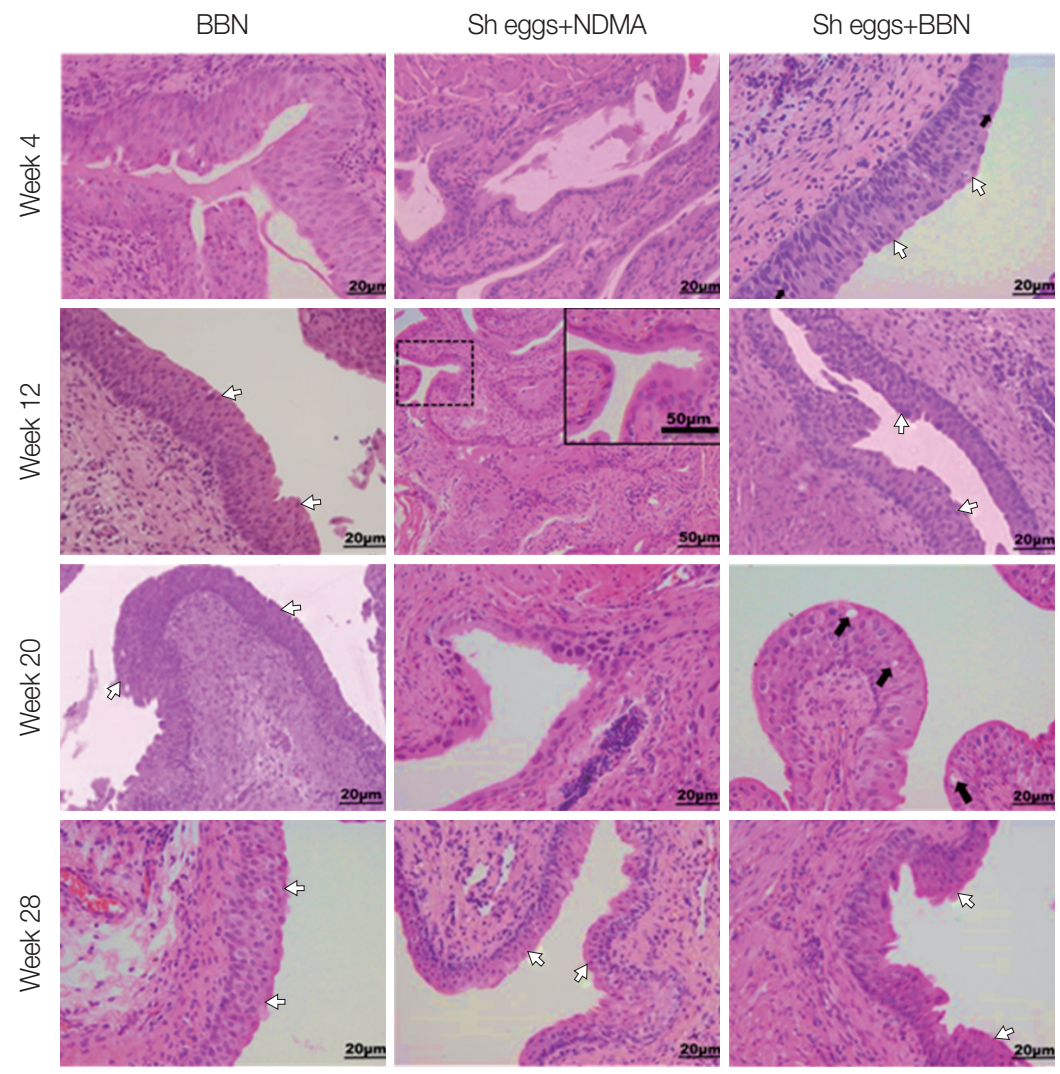

Fig. 3. Tissue sections of mouse urinary bladder showing major histopathological abnormalities (HE stain). BBN group, hyperplasia at week 4, hyperplasia and dysplasia at week 12, 20, and 28 (white arrows); Sh eggs+NDMA group, normal at week 4 and squamous metaplasia at week 12, and dysplasia and hyperplasia at week 20 and 28 (white arrows); Sh eggs +BBN group, hyperplasia and dysplasia (white arrows) at week 4 and 12, enlarged and pleomorphic cells (black arrows) and epithelial vacuolar change and hyperplasia and dysplasia (white arrows) at week 20 and 28, respectively (original magnification, $\times 400$ ). 

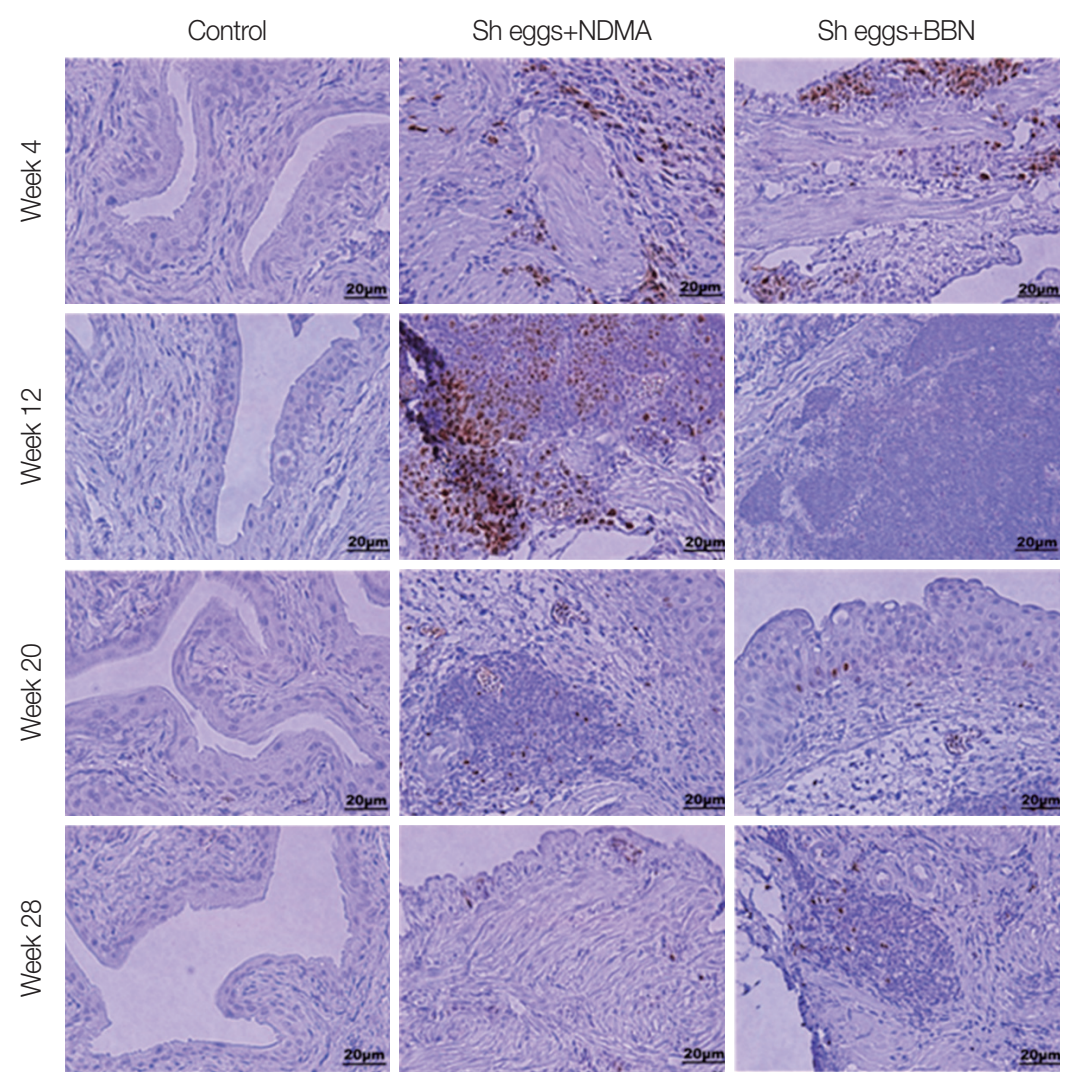

Fig. 4. Immunohistochemistry for staining of Ki-67 expression (brown color for positive cells) in the mouse urinary bladder wall.

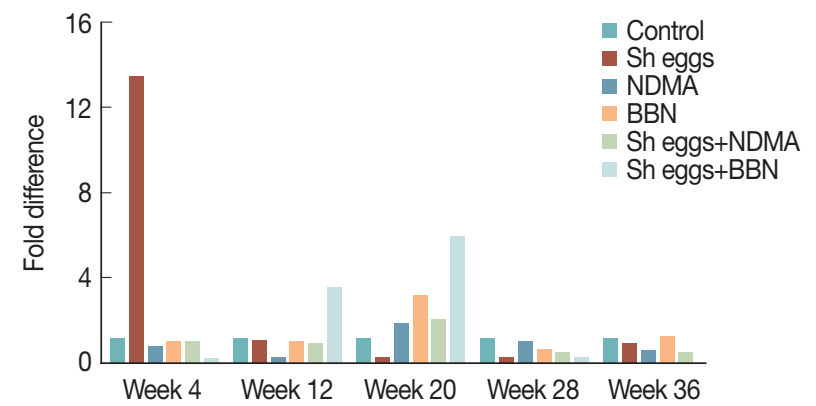

Fig. 5. Comparison of transcriptional expression of $p 53$ gene according to the treatments of $S$. haematobium eggs, NDMA, and BBN in different combination by qRT-PCR analysis. Saline injection was used as negative control. Relative mRNA expression was normalized to GAPDH expression levels, and shown by fold changes as mean \pm S.E.M $(n=2)$ fold differences at week 4, 12, 20,28 , and 36.

\section{Immunohistochemical results of $\mathrm{Ki}-67$ staining}

Immunohistochemical results of the present study showed positive Ki-67 expression in the urothelial epithelial cells of $S$. haematobium eggs+BBN group at week 20, while the remaining experimental groups showed either weak or no staining for urothelial Ki-67 staining (Fig. 4). However, there was strong

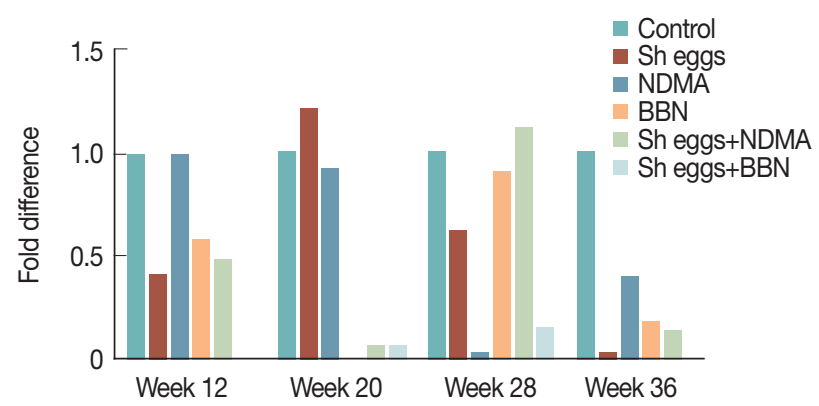

Fig. 6. Comparison of transcriptional expression of E-cadherin gene according to the treatments of $S$. haematobium eggs, NDMA, and BBN in different combination by qRT-PCR analysis. Saline injection was used as negative control. Relative mRNA expression was normalized to GAPDH expression levels, and shown by fold changes as mean \pm S.E.M $(n=2)$ fold differences at week 12, 20, 28, and 36.

staining for Ki-67 in lymphocytes in S. haematobium eggs+ NDMA and S. haematobium eggs+BBN groups at week 4 and 12 (Fig. 4). The intensity of lymphocyte staining slowly declined to moderate at week 20 and later. The control group did not show positive Ki-67 staining for both urothelial epithelial cells and in lymphocytes at all weeks of the experiment (Fig. 4). 

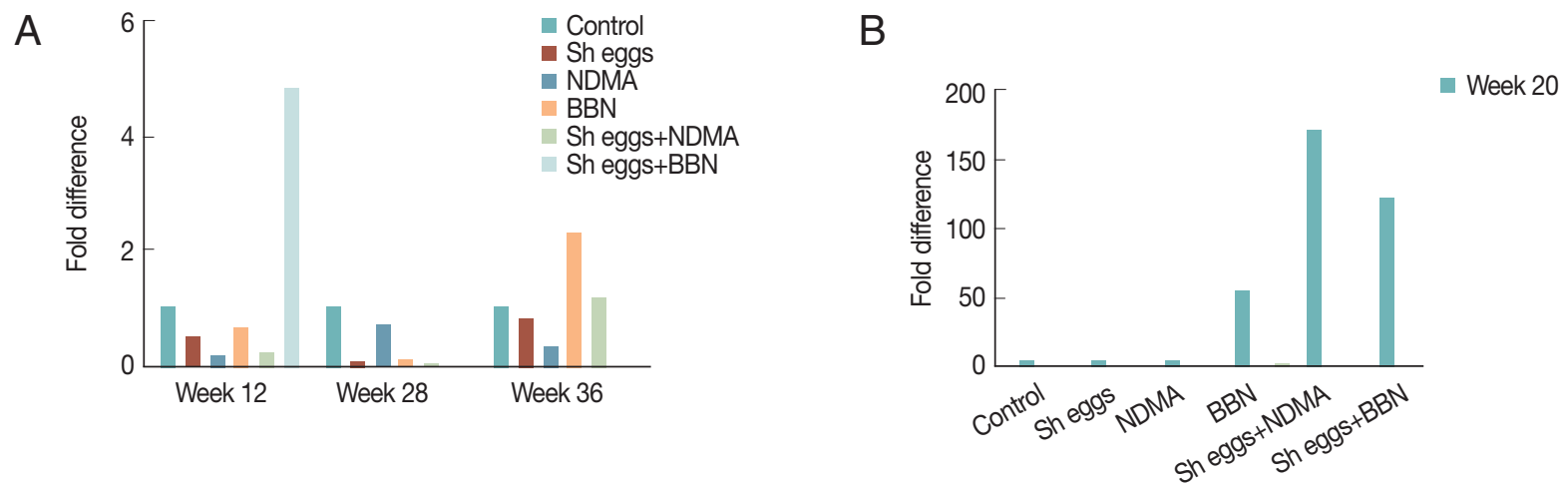

Fig. 7. Comparison of transcriptional expression of vimentin gene according to the treatments of S. haematobium eggs, NDMA, and $\mathrm{BBN}$ in different combination by qRT-PCR analysis. Saline injection was used as negative control. Relative mRNA expression was normalized to GAPDH expression levels, and shown by fold changes as mean \pm S.E.M $(n=2)$. (A) Fold differences at week 12,28 , and 36. (B) Fold differences at week 20.

\section{Expression of tumor suppressor and EMT marker genes}

Fold differences of $p 53$ relative to reference gene GAPDH significantly increased in $S$. haematobium eggs group at week 4 and S. haematobium eggs+BBN group at week 12 and 20 compared to the control, but no difference at the rest treatment weeks of the groups (Fig. 5).

Fold difference of E-cadherin was significantly low in BBN and $S$. haematobium eggs+BBN groups at all observation weeks. Reduction of E-cadherin at all observation weeks was observed except NDMA, S. haematobium eggs and S. haematobium eggs+NDMA groups at week 12, 20, and 28, respectively (Fig. $6)$, whereas vimentin showed a significant increase with peak at week 20 in BBN, S. haematobium eggs+NDMA and S. haematobium eggs+BBN groups. At week 12, only $S$. haematobium eggs+BBN group showed significant increase and BBN and $S$. haematobium eggs+NDMA group also increased at week 36 compared to the control (Fig. 7). Unfortunately, data for Ecadherin and vimentin at week 4 were not included due to missing of 2 samples from treatment groups.

\section{DISCUSSION}

The mice revealed delayed responses to stimuli and general lethargy among the S. haematobium eggs and NDMA or BBN treated groups. The strongly significant weight loss data of mice in S. haematobium eggs+NDMA and S. haematobium eggs $+\mathrm{BBN}$ groups may provide a clue for the synergistic impact of administration of S. haematobium eggs and nitrosamines on physical conditions.

The marked focal and dispersed tissue reactions and inflam- matory aggregates mainly among BBN, S. haematobium eggs+NDMA and $S$. haematobium eggs+BBN groups of the present study may give a substantial evidence for the effects of the eggs and nitrosamine chemicals. In addition to intense tissue reactions, histopathological abnormalities exhibited in aforementioned groups were also observed. Focal inflammation was observed in S. haematobium eggs+NDMA group at week 12 and 20. However, tissue reactions and inflammatory aggregates were slowly reduced in the treated groups in later weeks as can be observed in Fig. 2. Several studies showed that the chronic inflammatory processes induced by the eggs on the bladder wall produced toxic free radicals and $\mathrm{N}$-nitrosamines [10]. The epithelial vacuolar change, a whitish scaly appearance observed in S. haematobium eggs+BBN group at week 20 appeared to show vacuolar degeneration or transformation being on progress (Fig. 3).

Squamous metaplasia was observed in the S. haematobium eggs+NDMA group at week 12 and dysplasia and hyperplasia at week 20 and 28, respectively, post eggs injection and NDMA treatment (Fig. 3). Squamous metaplastic lesion was reported to have occurred secondary to inflammation initiated by urogenital schistosomiasis ultimately leading to transformation of UB to SCC [2]. Following squamous metaplasia, hyperplasia and dysplasia were observed among the group at week 20 and 28 but not at week 36 . Further cell proliferation or tissue reactions were seemingly more deteriorated at later weeks of observations. Furthermore, the chronological inconsistency of the tissue changes by weeks may have originated from individual variations of mice.

It was uncertain to estimate viability of the $S$. haematobium 
eggs and its impacts on UB pathology in the present study. The eggs were collected from infected humans and stored frozen at $-20^{\circ} \mathrm{C}$ for several months. The viability of the eggs may influence the pathogenesis in the UB but it is unknown. One study described viable and dead eggs were mixed in the intestinal tissue of $S$. mansoni infected mice [21]. The mice were kept for 69 days after the infection and about $1 / 4$ of eggs were found dead in the tissue. The viable eggs but not the dead ones may stimulate the tissue and cells more vigorously by secreting soluble molecules. Since the eggs are dying slowly in the tissue depending on the infection duration, and neoplasia is induced also in the chronic infection, the impact of egg viability must be an interesting topic of further research.

It has been reported that the expression of Ki-67, a nuclear cell proliferation marker, is strongly associated with tumor cell proliferation and growth, and is widely used in routine pathological investigation as a proliferation marker [22]. A number of diagnostic applications for $p K i-67$ have been described, where Ki-67 is significantly more expressed in malignant than in normal tissues [23]. Initiation and promotion of UB carcinogenesis by BBN was accompanied by increased cell proliferation in rodents owing to changes in the expression of genes involved in cell cycle control $[24,25]$. Previous studies reported no difference of Ki-67 expression between schistosomal and non-schistosomal bladder tumors [26]. Nevertheless, an increase in Ki-67-positive cells induced by BBN has recently been reported in female C57BL/6/c mice [14]. More recently, it has been reported that Ki-67 elimination might have minor effects on cell cycle exit and mitosis, and mammalian cells can nevertheless proliferate efficiently in the absence of detectable Ki-67 [27]. The present study findings on Ki-67 expression support its role in the prevailing urothelial transformation to hyperplasia, dysplasia, and epithelial vacuolar changes in $S$. haematobium eggs+BBN group mice treated with $S$. haematobium eggs and nitrosamines (Fig. 4).

Increase in the relative expression of $p 53$ might support cellular deregulation to be under progress in $S$. haematobium eggs+BBN group (Fig. 5). Studies showed that tumor suppressor gene is normally kept at very low levels and its level and activity increase dramatically in response to DNA damage, oncogenic stress, and other stimuli that are viewed by a cell as anomalous [28]. It has been reported that roughly $50 \%$ of chemically induced tumors in mice develop $p 53$ mutations [29], which are similar to those found in humans. Bladder cancers in schistosomiasis have also been reported to show pe- culiar profiles of $p 53$ and bcl-2 mutations compared to nonschistosome related bladder cancers [30]. A recent study reported that urothelial $p 53$ signaling indeed seemed to affect urothelial homeostasis during $S$. haematobium infection, albeit in a sex-specific manner [31].

An overlap of E-cadherin down regulation and vimentin upregulation in S. haematobium eggs+BBN group at week 12 and 20 of the present study is likely linked to aberrant situation occurring during EMT (Fig. 6). Remarkably, Ki-67 positivity and histopathological abnormalities, including hyperplasia, dysplasia, and epithelial vacuolar change synchronized with the abrupt increase of vimentin in S. haematobium eggs+BBN groups appear to be a presumptive evidence for urothelial transformation under progress. Furthermore, profound tissue reactions with Ki-67 expression and sharp increase of vimentin exclusively among $S$. haematobium eggs+BBN group at week 12 can also be another suggestive clue for pre-neoplastic changes. Similarly, the spike increase of vimentin expression in BBN and S. haematobium eggs+NDMA groups at week 20 coupled with histopathological and Ki-67 data at the same observation time may also implicate the ongoing pre-malignant development. E-cadherin is a transmembrane glycoprotein that mediates calcium-dependent, homophilic cell-cell adhesion in all epithelial tissues. It is the main mediator of cell-cell adhesion in epithelial tissues and expressed by most normal epithelial cells [32]. Vimentin has been reported to play important roles in cell adhesion, migration, and signaling [33]. Recent studies showed that the expression of vimentin was observed in $43 \%$ of bladder cancers, whereas it was not expressed or found negative in all normal urothelial cells [34]. It has been implicated that loss of E-cadherin and acquisition of vimentin are 2 critical steps in EMT, where epithelial cells undergo a transformation into spindle-shaped mesenchymal cells, acquire malignant properties, and become more migratory and invasive [35]. The present findings in the $S$. haematobium eggs+NDMA and $S$. haematobium eggs+BBN groups confirmed that the urothelial cells stimulated by $S$. haematobium eggs and nitrosamine chemicals of NDMA or BBN were slowly transformed into neoplastic cells. A longer experiment may reveal neoplastic cells.

The present study had some limitations. The number of $S$. haematobium eggs must be at least 2,000 per mouse for better outcomes. Relatively short duration of the experiment may be another possible limitation since oncogenesis usually takes a long period. 
In conclusion, the pre-neoplastic changes of UB cells were observed in mice of $S$. haematobium eggs and nitrosamine groups but not in $S$. haematobium eggs alone or nitrosamine alone groups. The eggs and nitrosamines synergistically transform UB epithelial cells into squamous metaplasia and dysplasia. The increased Ki-67 expression may be correlated with the transformation. Also the transformation is correlated with expression of the oncogenes in treated groups; marked decrease in E-cadherin at almost all weeks and increase in $p 53$ and vimentin at specific weeks for $S$. haematobium eggs and $S$. haematobium eggs+BBN groups were observed. The present study provided a possibility of the mouse model for bladder cancer study by $S$. haematobium .

\section{ACKNOWLEDGMENT}

This work was supported by the Education and Research Encouragement Fund of the Seoul National University Hospital (2016).

\section{CONFLICT OF INTEREST}

We have no conflict of interest related to this work.

\section{REFERENCES}

1. IARC Working Group on the Evaluation of Carcinogenic Risks to Humans. Biological agents. Volume 100 B. A review of human carcinogens. IARC Monogr Eval Carcinog Risks Hum 2012; 100(Pt B): 1-441.

2. Rausch S, Hofmann R, von Knobloch R. Nonbilharzial squamous cell carcinoma and transitional cell carcinoma with squamous differentiation of the lower and upper urinary tract. Urol Ann 2012; 4: 14-18.

3. Mostafa HM, Sheweita SA, O'Connor PJ. Relationship between schistosomiasis and bladder cancer. Clin Microbiol Rev 1999; 12: 97-111.

4. Shirahama T, Sakakura C. Overexpression of cyclooxygenase-2 in squamous cell carcinoma of the urinary bladder. Clin Cancer Res 2001; 7: 558-561.

5. Heyns CF, van der Merwe A. Bladder cancer in Africa. Can J Urol 2008; 15: 3899-3908.

6. Felix AS, Soliman AS, Khaled H, Zaghloul MS, Banerjee M, ElBaradie M, El-Kalawy M, Abd-Elsayed AA, Ismail K, Hablas A, Seifeldin IA, Ramadan M, Wilson ML. The changing patterns of bladder cancer in Egypt over the past 26 years. Cancer Causes Control 2008; 19: 421-429.

7. Shiff C, Naples JM, Isharwal S, Bosompem KM, Veltri RW. Non- invasive methods to detect schistosome-based bladder cancer: is the association sufficient for epidemiological use? Trans R Soc Trop Med Hyg 2010; 104: 3-5.

8. Rambau PF, Chalya PL, Jackson K. Schistosomiasis and urinary bladder cancer in North Western Tanzania: a retrospective review of 185 patients. Infect Agent Cancer 2013; 8: 19.

9. Botelho MC, Figueiredo J, Alves H. Bladder cancer and urinary Schistosomiasis in Angola. J Nephrol Res 2015; 1: 22-24.

10. Nweke CP, Shiff CJ, Okudo JC. The etiological association between Schistosoma hematobium infestation and bladder cancer in Africa and the Middle East. Peak J Public Health Manag 2015; 3: 10-17.

11. Kuntz RE, Cheever AW, Myers BJ, Young SW, Moore JA. Calcification of the bladder and papillary tumours of the bladder and ureters in gibbons (Hylobates lar) infected with Schistosoma haematobium (Iran). Trans R Soc Trop Med Hyg 1975; 69: 494-502.

12. Hicks RM, James C, Webbe G. Effect of Schistosoma haematobium and N-butyl-N-(4-hydroxybutyl)nitrosamine on the development of urothelial neoplasia in the baboon. Br J Cancer 1980; 42: $730-755$.

13. George SK, Tovar-Sepulveda V, Shen SS, Jian W, Zhang Y, Hilsenbeck SG, Lerner SP, Smith CL. Chemoprevention of BBN-Induced Bladder Carcinogenesis by the Selective Estrogen Receptor Modulator Tamoxifen. Transl Oncol 2013; 6: 244-255.

14. Henriques A, Arantes-Rodrigues R, I Faustino-Rocha A, I TeixeiraGuedes C, Pinho-Oliveira J, Talhada D, H Teixeira J, Andrade A, Colaço B, N Paiva-Cardoso M, J Pires M, Mvd Ferreira A, M Nunes F, A Oliveira P. The effects of whole green tea infusion on mouse urinary bladder chemical carcinogenesis. Iran J Basic Med Sci 2014; 17: 145-148.

15. Fu CL, Apelo CA, Torres B, Thai KH, Hsieh MH. Mouse bladder wall injection. J Vis Exp 2011; 12: e2523.

16. Fu CL, Odegaard JI, Herbert DR, Hsieh MH. A novel mouse model of Schistosoma haematobium egg-induced immunopathology. PLoS Pathog 2012; 8: e1002605.

17. Eble JN, Sauter G, Epstein JI, Sesterhenn IA. Pathology and genetics of tumors of the urinary system and male genital organs. WHO, IARC Press; 2004.

18. Madka V, Zhang Y, Li Q, Mohammed A, Sindhwani P, Lightfoot S, Wu XR, Kopelovich L, Rao CV. p53-stabilizing agent CP-31398 prevents growth and invasion of urothelial cancer of the bladder in transgenic UPII-SV40T mice. Neoplasia 2013; 15: 966-974.

19. Liang Z, Xie W, Wu R, Geng H, Zhao L, Xie C, Li X, Zhu M, Zhu W, Zhu J, Huang C, Ma X, Wu J, Geng S, Zhong C, Han H. Inhibition of tobacco smoke-induced bladder MAPK activation and epithelial-mesenchymal transition in mice by curcumin. Int J Clin Exp Pathol 2015; 8: 4503-4513.

20. Schmittgen TD, Livak KJ. Analyzing real-time PCR data by the comparative C(T) method. Nat Protoc 2008; 3: 1101-1108.

21. Espíndola MS, Frantz FG, Soares LS, Masson AP, Tefé-Silva C, Bitencourt CS, Oliveira SC, Rodrigues V, Ramos SG, Silva CL, Faccioli LH. Combined immunization using DNA-Sm14 and DNAHsp65 increases CD8+ memory T cells, reduces chronic pathol- 
ogy and decreases egg viability during Schistosoma mansoni infection. BMC Infect Dis 2014; 14: 263.

22. Ding W, Gou Y, Sun C, Xia G, Wang H, Chen Z, Tan J, Xu K, Qiang D. Ki-67 is an independent indicator in non-muscle invasive bladder cancer (NMIBC); combination of EORTC risk scores and $\mathrm{Ki}-67$ expression could improve the risk stratification of NMIBC. Urol Oncol 2014; 32: 42. e13-19.

23. Hu HY, Liu H, Zhang JW, Hu K, Lin Y. Clinical significance of Smac and Ki-67 expression in pancreatic cancer. Hepatogastroenterology 2012; 59: 2640-2643.

24. Tyagi A, Raina K, Singh RP, Gu M, Agarwal C, Harrison G, Glode LM, Agarwal R. Chemopreventive effects of silymarin and silibinin on N-butyl-N-(4-hydroxybutyl) nitrosamine-induced urinary bladder carcinogenesis in male ICR mice. Mol Cancer Ther 2007; 6: 3248-3255.

25. Williams PD, Lee JK, Theodorescu D. Molecular credentialing of rodent bladder carcinogenesis models. Neoplasia 2008; 10: 838846.

26. Abdulamir AS, Hafidh RR, Kadhim HS, Abubakar F. Tumor markers of bladder cancer: the schistosomal bladder tumors versus non-schistosomal bladder tumors. J Exp Clin Cancer Res 2009; 28: 27.

27. Sobecki M, Mrouj K, Camasses A, Parisis N, Nicolas E, Llères D, Gerbe F, Prieto S, Krasinska L, David A, Eguren M, Birling MC, Urbach S, Hem S, Déjardin J, Malumbres M, Jay P, Dulic V, Lafontaine DLj, Feil R, Fisher D. The cell proliferation antigen Ki67 organizes heterochromatin. ELife 2016; 5: e13722.
28. Lozano G. Mouse models of $p 53$ functions. Cold Spring Harb Perspect Biol 2010; 2: a001115.

29. Yamamoto $S$, Chen T, Murai T, Mori S, Morimura K, Oohara T, Makino S, Tatematsu M, Wanibuchi H, Fukushima S. Genetic instability and $p 53$ mutations in metastatic foci of mouse urinary bladder carcinomas induced by N-butyl-N-(4-hydroxybutyl)nitrosamine.. Carcinogenesis 1997; 18: 1877-1882.

30. Helal Tel A, Fadel MT, El-Sayed NK. Human papilloma virus and p53 expression in bladder cancer in Egypt: relationship to schistosomiasis and clinicopathologic factor. Pathol Oncol Res 2006; 12: $173-178$.

31. Honeycutt J, Hammam O, Hsieh MH. Schistosoma haematobium egg-induced bladder urothelial abnormalities dependent on $p 53$ are modulated by host sex. Exp Parasitol 2015; 158: 55-60.

32. Oda H, Takeichi M. Evolution: structural and functional diversity of cadherin at the adherens junction. J Cell Biol 2011; 193: 1137-1146.

33. Ivaska J, Pallari HM, Nevo J, Eriksson JE. Novel functions of vimentin in cell adhesion, migration, and signaling. Exp Cell Res 2007; 313: 2050-2062.

34. Ding X, Wang Y, Ma X, Guo H, Yan X, Chi Q, Li J, Hou Y, Wang C. Expression of HMGA2 in bladder cancer and its association with epithelial-to-mesenchymal transition. Cell Prolif 2014; 47: 146151.

35. Thiery JP, Acloque H, Huang RY, Nieto MA. Epithelial-mesenchymal transitions in development and disease. Cell 2009; 139: 871-890. 